\title{
Detection of Biofilm Formation of Klebsiella Pneumoniae Isolated from Medical Devices at the University Hospital of Tlemcen, Algeria
}

\author{
Type of article: Conference abstract \\ Samia Bellifa1, Hafida Hassaine1, Ibrahim Benamar1, Ibtissem Kara Terki1, Imane M'hamedi1, \\ Merieme Lachachi1, Sara Gaouar 1, Christiane Forestier2 \\ 1Laboratoire de Microbiologie Appliquée à l'agroalimentaire au Biomédical et à l'environnement \\ (LAMAABE), Université de Tlemcen, Algeria \\ 2Clermont Université, UMR CNRS 6023 Laboratoire Microorganismes: Génome Environnement \\ (LMGE), Université d'Auvergne, Clermont-Ferrand, France \\ Corresponding Author: samia.bellifa@yahoo.fr
}

\begin{abstract}
:
Background: Klebsiella pneumoniae is a major cause of community-acquired and nosocomial infections. This germ is responsible for acute and chronic infections, most of which are due to its ability to adhere to medical implants and form a biofilm. The objective of this work is to study the interaction between clinical isolates of $K$. pneumoniae and abiotic surfaces (medical devices) and some factors influencing biofilm formation.

Methods: Over a period of 2 years, 115 strains of $K$. pneumoniae were isolated from medical devices CHU Tlemcen, most of which had a high level of resistance to cephalosporins $1^{\text {st }}, 2^{\text {nd }}$, and $3^{\text {rd }}$ generation. Their capacity to form biofilm was assessed using 3 techniques: TCP, TP, and RCA. We determined in vitro the effects of three antimicrobial agents against planktonic and biofilm forms of K. pneumoniae. The presence of MrkD genes was detected by polymerase chain reaction (PCR).

Results: According to the studied (TCP, TP, RCA) strains of $K$.pneumoniae isolated from urinary catheters have proved very good, forming the biofilm to those isolated from other medical devices. 24 of 115 isolated strains showed a clear difference in antibiotic susceptibility between planktonic populations and biofilm populations. They were 10-20 times higher. All strains presented a highly hydrophilic character and adhesion 2-10 times greater in PVC with respect to glass support. The MrkD gene (detected by PCR) responsible for biofilm formation was found in 22 strains of $K$. pneumoniae, which may explain their adhesion and therefor their pathogenicity.

Conclusion: Our results show the great ability of K.pneumoniae strains to form a biofilm on medical devices, and the isolates were at least 10 times more resistant than their planktonic counterparts. In addition, we showed that the presence of type 3-encoding gene $m r k D$ was associated with high adhesion indexes.
\end{abstract}

Keywords: Biofilm, Klebsiella Pneumoniae, Antibiotic Susceptibility, Urinary Catheter, Medical Devices.

\section{Conflict of interest statement}

This article is a conference abstract presented at the International Congress on Health Sciences and Medical Technologies, Tlemcen Algeria 5-7 December 2019, ICHSMT' 19.

\section{Authors' biography}

No Biography. 
Medical Technologies Journal, Volume: 4, Issue: 1, January-March 2020, Pages: 518-519. Doi :

https://doi.org/10.26415/2572-004X-vol4iss1p518-519

\section{References}

No references 